\title{
Fire Detection Methods Based on Various Color Spaces and Gaussian Mixture Models
}

\author{
Amr A. Munshi ${ }^{*}$ \\ 1 Computer Engineering Department, Umm Al-Qura University, Saudi Arabia \\ * Corresponding author's email: aaamunshi@uqu.edu.sa
}

\begin{abstract}
Fire disasters are very serious problems that may cause damages to ecological systems, infrastructure, properties, and even a threat to human lives; therefore, detecting fires at their earliest stage is of importance. Inspired by the technological advancements in artificial intelligence and image processing in solving problems in different applications, this encourages adopting those technologies in reducing the damage and harm caused by fire. This study attempts to propose an intelligent fire detection method by investigating three approaches to detect fire based on three different color models: RGB, YCbCr, and HSV were presented. The RGB method is applied based on the relationship among the red, green and blue values of pixels in images. In the $\mathrm{YCbCr}$ color model, image processing and machine learning techniques are used for morphological processing and automatic recognition of fire images. In turn, for HSV, supervised machine learning techniques are adopted, namely decision rule and Gaussian mixture model (GMM). Further, the expectation maximization (EM) algorithm was deployed for the GMM parameters estimation. The three proposed models were tested on two data sets, one of which contains fire images, the other consists of non-fire images with some having fire-like colors to test the efficiency of the proposed methods. The experimental results showed that the overall accuracies on two data sets for the RGB, $\mathrm{YCbCr}$, and HSV methods were satisfactory and were efficient in detecting the outdoor and indoor fires.
\end{abstract}

Keywords: fire detection; fire pixels; fire-like pixels; color models; Gaussian mixture models.

\section{INTRODUCTION}

Fire disasters are serious problems around the world. In order to predict fire disasters, various methods have been used to detect fires, such as fire watch towers, physical sensors, satellite and aerial monitoring, and video and digital image analysis. The reliability of fire watch tower depends greatly on the operator's assessment. Physical sensors, which are often based on particle sampling, temperature sampling, relative humidity sampling, air transparency testing, and smoke analysis, can cause false alarms, for example, individual smoking nearby or vapor from the cooking process. Moreover, reliability of this fire detection system depends on the distribution of sensors; and the fire location, its size as well as growing rate cannot be determined. Therefore, this kind of detection is only suitable to close and small areas. Meanwhile, the satellite and aerial monitoring system is very expensive and can be activated when the fire has already spread significantly.

There has been a continuous interest in conducting the studies related to fire detection based on video and image analysis. Most of the work in fire detection in color images and videos are based on setting color rules. [1, 2] proposed a method of developing a set of rules for fire pixel classification based on video image processing. The RGB color system was used to extract fire pixels and smoke pixels, which are then verified if they are real fire pixels or fire-like pixels. $[3,4]$ attempted to detect fires and flames by processing the video data generated by an ordinary camera monitoring a scene. The fire pixels are determined by ordinary motion, RGB color clues, and video analysis in the wavelet domain. 
[5] proposed an approach to detect fires in real time by combining the foreground object information with color pixels statistics of fire based on the RGB color system. Other methods utilize threshold selection and image feature analysis for early fire detection [6]. An adaptive background subtraction algorithm was developed to extract the foreground information, then this foreground region was verified to determine if it is a fire candidate or not. The algorithm was the developed [7] utilizing a $\mathrm{YCbCr}$ color space, since it can separate the illumination information from chrominance more efficiently than other color space. [8] used flame contours for early fire detection from video sequences. The process of fire detection consists of candidate fire frame selection, flame region selection, and flame contour-based fire decision. [9] utilized the RGB color space to develop a set of rules to classify the fire pixels from images. Meanwhile, [10] proposed an approach in which the statistical fire color model (RGB) and sequential pattern mining technology are combined to detect fire in images.

Many studies have been conducted on detecting fire using the $\mathrm{YCbCr}$ color model as well. In a research conducted by [7], the YCbCr color system was utilized. The advantage of using such color system is the ability of separating the luminance from the chrominance more effectively than other color spaces. Then a generic chrominance model was proposed for flame pixel classification. This further alleviates the harmful effects of changing illumination and also detects fire more efficiently; moreover, higher accuracy rates were achieved by utilizing the $\mathrm{YCbCr}$ color system over the RGB color system. [11] also performed a research based on the color model approach, to classify a pixel to be fire the model identifies seven rules. If a pixel satisfies those seven rules, then it is classified as fire. In this method, two color systems namely, RGB and $\mathrm{YCbCr}$ were utilized in the classification process. However, this approach was limited to detecting forest fires, which hinders the usage of this approach.

Due to developments in digital image and video processing, also inspired by the artificial intelligence capabilities, computer vision-based fire alarm systems tend to replace the conventional

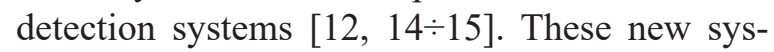
tems can cover larger detection areas with higher reliability. Thus, exploring the approaches to detect fire based on color systems and the application of machine learning techniques is of interest.
The remainder of the paper is structured as follows. Section 2 presents the methodology to extract fire pixels from images; further, the results on a real-world dataset are presented. Section 3 presents a fire detection algorithm based the $\mathrm{YCbCr}$ color space and the results are evaluated on real images. The details of detecting fire regions based on an HSV color model and the decision rules to validate fire utilizing the Gaussian mixture model (GMM) are presented in Section 4. Finally, the conclusions are drawn.

\section{The RGB Based Method}

By studying the visual features of fire, it is can be observed that fires have unique visual features. These features can be divided into static and dynamic characteristics, which are both used in the classification of fire pixels. In terms of static characteristics, flames often have reddish colors and they will vary with an increase in temperature. When the fire is in its early stage, its temperature is low and the colors range from red to yellow. However, when the fire develops and reaches higher temperature, its color may turn into white. This means that a low-temperature flame emits a light with high saturation, whereas a high-temperature flame emits a low-saturation light. In addition, the saturation in the day-time or with background illumination is stronger than that of during the night-time or without light source. Accordingly, three color spectra of the segmented fire region exist namely, white-yellow, orange, and red. In terms of dynamic characteristics, the shape of the flame varies with time, because it could be affected by the wind. Furthermore, the fire becomes larger and larger if it is not extinguished; therefore, the area of the flame also varies. These properties are often utilized in the separation of the foreground (the flame) from the background in order to increase the reliability of the method.

In this section, the static characteristics of fires in the RGB color space to determine fire pixels in color images are deployed.

\section{The RGB Color Space}

The RGB color model [13] is based on a Cartesian coordinate system. The RGB model forms its gamut from the primary additive colors of red, green and blue. The combination of red, green and blue light in the same amounts forms white. Computers generally display RGB images using 24-bit 
color. In the 24-bit RGB color model, there are 256 variations for each of the additive colors of red, green and blue. Therefore, there are 256 reds x 256 greens $\times 256$ blues $=16,777,216$ possible colors in the 24-bit RGB color model. In this color model, colors are represented by varying intensities of red, green and blue light. The intensity of each of the red, green and blue components is represented on a scale from 0 to 255 with 0 being the least intensity to the maximum intensity of 255 .

\section{The RGB Color Space Based Algorithm}

The most important step of the implementation is to train data so that a model is constructed. This model should be able to verify if there were flames in the input images data. The training data, including the threshold (upper-bounds and lowerbounds) for the relationship of RGB values, were determined from the histograms of the region of interest (ROI) containing flame in each image. A dataset consisting of seventy images containing flames was utilized. Then, for this dataset, the ROI is determined and separated into three different color channels. Further, their histograms are studied in order to find the correlation among the RGB values and HSI values. The maximum, minimum, and mean values of each pixel of each color channel were observed. From the dataset, seventy sets of RGB were extracted. The histograms from those images consisting of flames

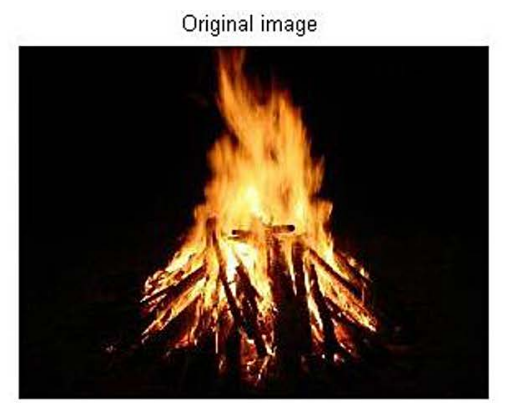

(a) The image

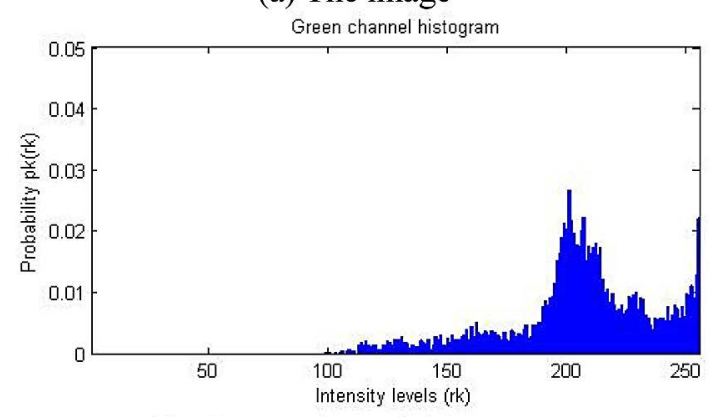

(c) Green channel histogram were investigated to find out if any patterns exist. Figure 1 shows an example of a set of histograms obtained by extracting the ROI over the flames.

$$
R(x, y)>G(x, y)>B(x, y)
$$

where: $(x, y)$ is the coordination of the pixel; $R, G$, $B$ are the intensities of the pixel at location $(\mathrm{x}, \mathrm{y})$ respectively. For a fire pixel, the red-channel pixel has a higher value than the green channel pixel and the greenchannel pixel value is greater than that of the blue channel.

Furthermore, the red-channel value and the green-channel value of a fire pixel are greater than 190 and 90, respectively; in turn, the bluechannel value of the same pixel is less than 140 . This introduces the second condition:

$R(x, y)>190 ; G(x, y)>90 ; B(x, y)<140(2)$

However, fire-like colors are sometimes misclassified as fire, leading to false alarms. As mentioned previously, both luminance (intensity) and chrominance changes in direct proportion to illumination. When a camera captures an image, the illumination it obtains from a flame is higher than that of a fire-like object, such as orange, rose, and red tiles. Therefore, the luminance and chrominance of flame are higher than those of fire-like objects. In order to overcome this, chromaticity

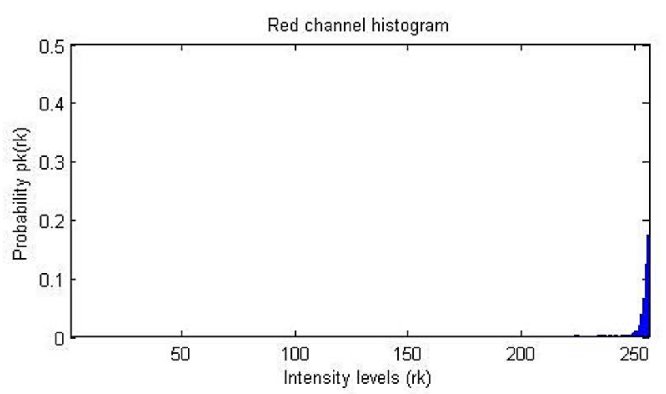

(b) Red channel histogram

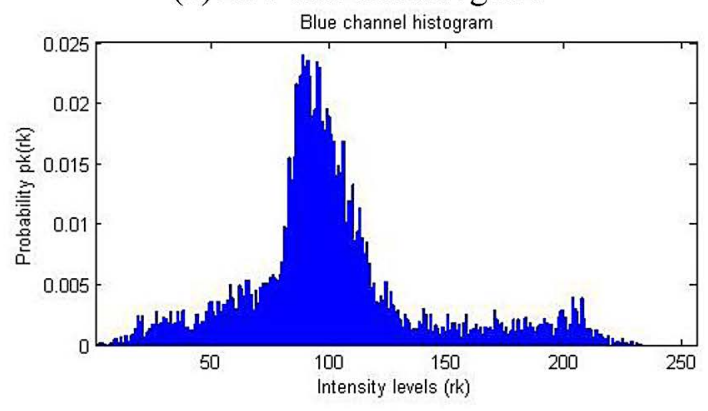

(d) Blue channel histogram

Fig. 1. Histograms of a region over the flame 
is deployed. As the RGB colors contain the luminance and the chrominance of the pixels, it is desired to separate them. In order to accomplish this, two luminance and chrominance separation techniques are mentioned in the following sections. The third observed condition for classifying color-based fire should satisfy:

$$
\begin{array}{r}
0.10 \leq \frac{G(x, y)}{R(x, y)+1} \leq 1.0 \\
0.10 \leq \frac{\mathrm{B}(\mathrm{x}, \mathrm{y})}{\mathrm{R}(\mathrm{x}, \mathrm{y})+1} \leq 0.85 \\
0.10 \leq \frac{B(x, y)}{G(x, y)+1} \leq 0.85
\end{array}
$$

Accordingly, the pixels in images that satisfy the three inequalities (1), (2), and (3), are considered fire pixels. The flowchart of the presented method based on the RGB color model is illustrated in Figure 2.

In this method, an image is the input. The image is then separated into three color channels, namely the Red channel, the Green channel, and the Blue channel. Next, every pixel of the image is scanned to verify if it is a candidate fire pixel. If the RGB values of the scanned pixel satisfy the three inequalities, the pixel is classified as a fire pixel and labelled 1 ; otherwise, it is labelled 0 and the next pixel (if any) is scanned. Then, the binary image is morphologically processed to remove small objects and fill holes to improve the shape of the flame. Finally, once all the pixels of the image are scanned, if the number of detected fire pixels exceeds the threshold, the fire alarm is activated. In this setting, the threshold of fire pixels was set to 50 , as small blobs of fire can contain over 50 pixels. The purpose of setting this threshold is to reduce the number of false alarms due to fire-like colors and hence increase the reliability of the method.

\section{Results of RGB Color Space Based Algorithm}

In order to verify the method, one hundred and forty images were collected, seventy of which contain fires or flames, the remaining images do not contain fire, but some images contain

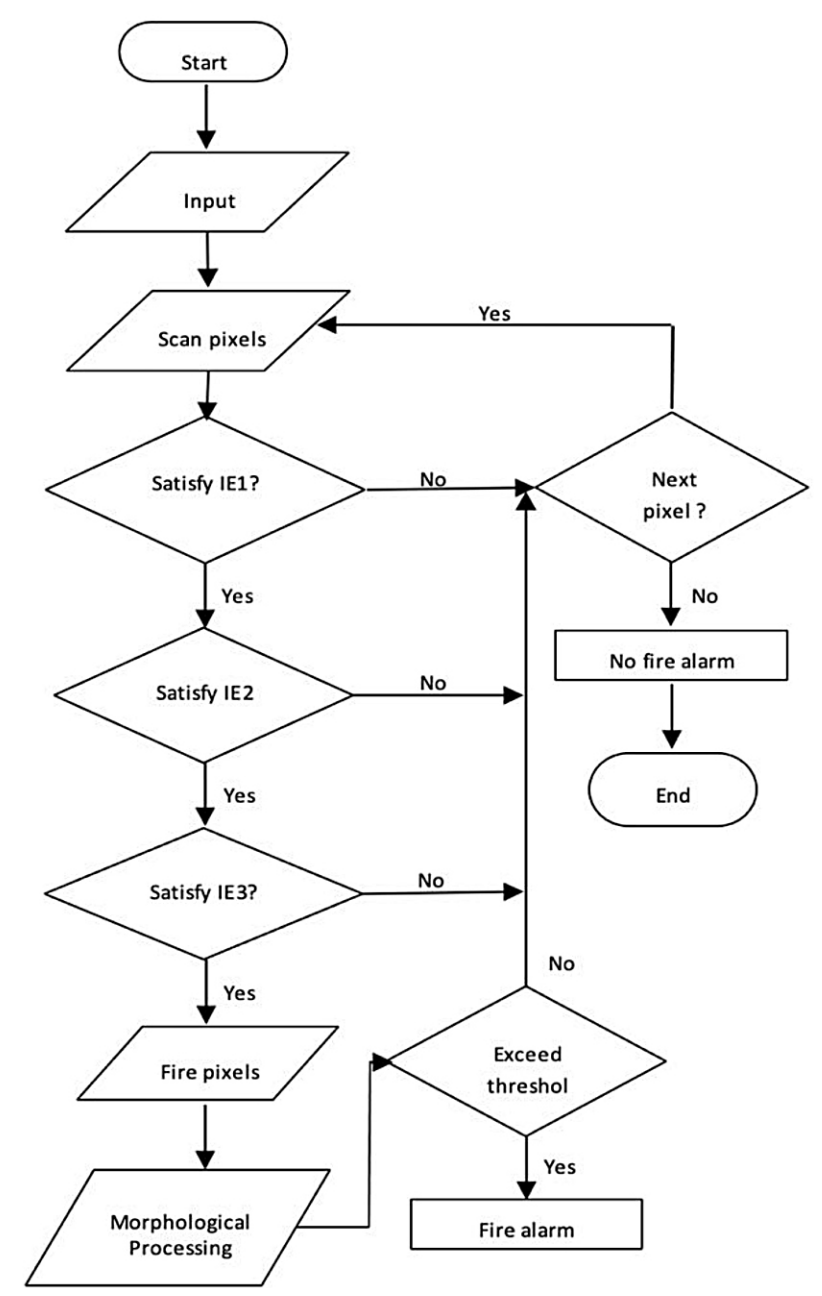

Fig. 2. Flowchart of the proposed method based on the RGB color model 
fire-like colors. Each image was scanned using inequalities (1), (2), and (3) to determine if fire pixels exist in the image. Figure 3 shows fire pixel segmentations in the binary images when inequalities (1), (2) and (3) were applied. Figure 3(a) shows the original image; Figure 3(b) shows the binary image, in which the pixels satisfying inequality (1) are mapped to white; Figure 3 (c) demonstrates the resulting binary image when rules (1) and (2) were applied to the original image. The final image in Figure 3(d) is the result of the simultaneous application of rules (1)-(3), and morphological processing to segment fire pixels in the original image. The morphological processing removed objects with the number of pixels less than 50 and filled the other objects with additive pixels. It can be clearly seen that the more rules applied to the original image, the more

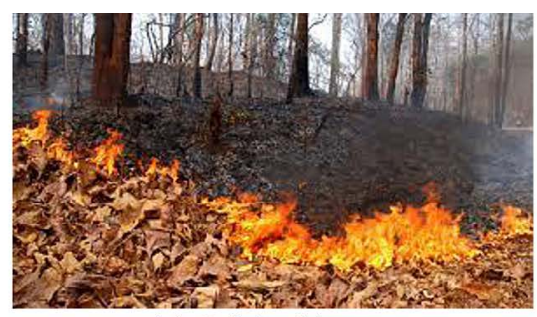

(a) Original image

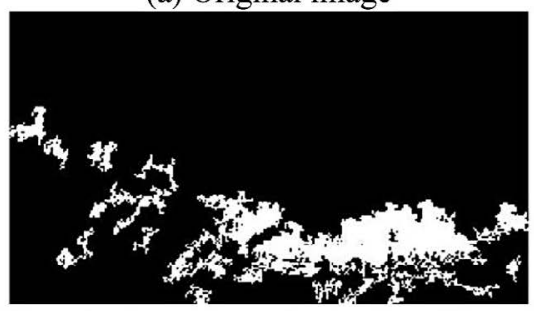

(c) Fire pixel segmentation using (1) and (2) similar the segmented flame to original flame. As can be seen from Figure 4, the dried leaves on the ground have a similar color to that of the flame; however, the image was not classified as a fire image because the three rules and morphological processing were unsatisfied.

However, there were some cases in which the images containing no flame but fire-like colors can be segmented as fire, even though three rules were all applied. Figure 4 shows the results when rule (1), rules (1) + rule (2), rule (1) + rule (2) + rule (3) were applied, respectively.

When the first set of images were tested, which contains seventy images of fire, $100 \%$ of the images were classified as fire. This means that the accuracy is $100 \%$. However, when the algorithm was tested on the second set of data including some images having fire-like colors, false

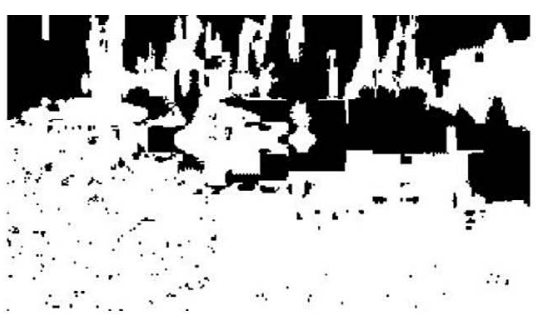

(b) Fire pixel segmentation using (1)

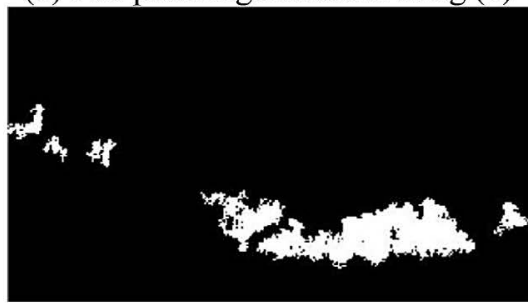

(d) Fire pixel segmentation using (1), (2), and (3)

Fig. 3. Fire pixel segmentation

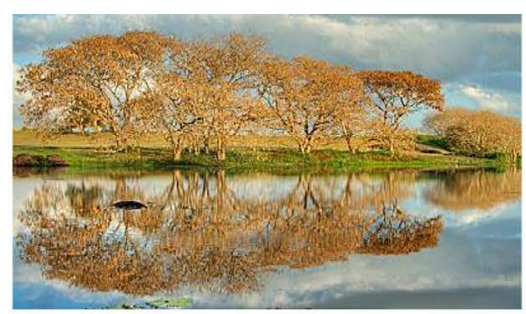

(a) Original image

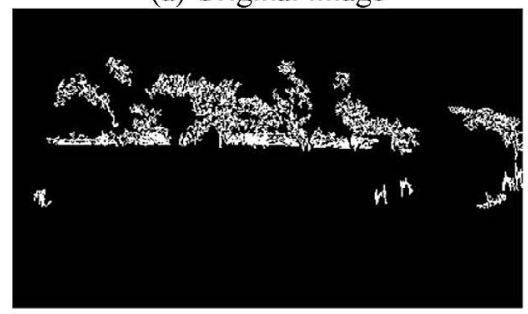

(c) Fire pixel segmentation using (1) and (2)
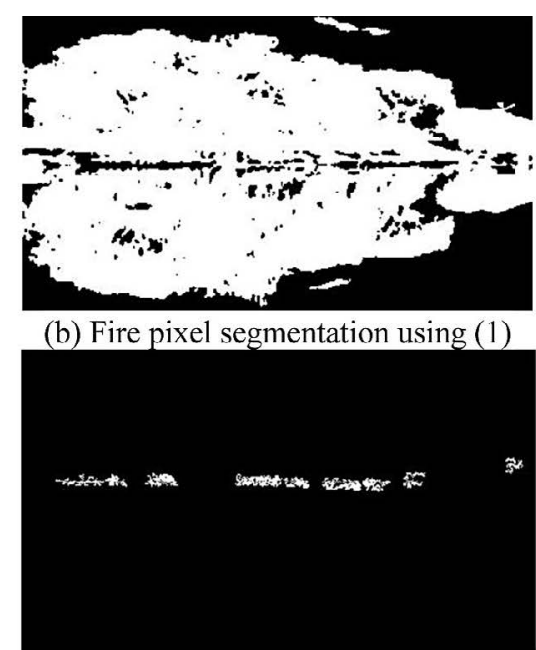

(d) Fire pixel segmentation using (1), (2), and (3)

Fig. 4. Fire pixel segmentation 
positive classifications appeared (false alarms), which accounted for approximately $27 \%$. After training the model, thirty other images with flames were randomly collected from the Internet and fed into the program for testing. All images were correctly classified.

$$
\text { accuracy }=\frac{\text { correct predictions }}{\# \text { of images }} \times 100 \%
$$

The RGB color model is very popular for color cameras, color monitors, and color images. From the results it might be thought that this method would offer extremely accurate results. However, it was observed that false alarms occur in $27 \%$ for the cases of fire-like colors. It was difficult to distinguish between the fire pixels and the firelike pixels in the RGB color model. One static characteristic that can assist in overcoming this problem is the luminance level of the pixel being considered. Unfortunately, the RGB color model represents both luminance and chrominance.

In order to obtain a higher accuracy, color and motion information can be combined. For instance, in order to distinguish between the fire pixels and fire-like pixels, the dynamic characteristics such as the motion, the changing area and other features of the flame region can be deployed to separate the foreground of the flame from the background.

This approach did not mention smoke detection. In many cases, fire in its early stages there may be no flames, but smoke is captured. Other criteria for smoke detection are needed due to the different color characteristics from those of the fire pixels.

\section{The YCbCr Based Method}

One of the primary color systems used to represent digital video component is the $\mathrm{YCbCr}$ color system. It represents color as the brightness and two-color difference signals, where $\mathrm{Y}$ is the luminance, $\mathrm{Cb}$ is blue minus luminance $(\mathrm{B}-\mathrm{Y})$ and $\mathrm{Cr}$ is red minus luminance (R-Y). $\mathrm{Y}$ is defined to have a nominal of 8-bit range of 16 to 235 , but $\mathrm{Cb}$ and $\mathrm{Cr}$ have a nominal range of 16 to 240 . An advantage of the $\mathrm{YCbCr}$ color system is separating the luminance from the chrominance more effectively than other color systems. For this reason, it is chosen to experiment its effectiveness on distinguishing fire pixels.

The performance of the proposed algorithm using the $\mathrm{YCbCr}$ color system to distinguish the fire pixels is tested on the same datasets using for verifying the RGB color system, two sets of images, one of which contains fire regions and the other containing different images with some of having fire-like regions.

\section{The YCbCr Color Space Based Algorithm}

In order to classify a pixel as a fire pixel utilizing the $\mathrm{YCbCr}$ color system, Figure 5 shows the flow chart of the proposed algorithm. It is based on the characteristics of the $\mathrm{YCbCr}$ color system. This $\mathrm{YCbCr}$ based approach has been followed due to its simplicity, effectiveness and wide range of use. In order to classify a pixel as a fire pixel, there are three rules that have to be satisfied. If a pixel satisfies these three rules, then it is considered to be a fire pixel.

\section{Rule 1: YCbCrIntensity Values}

After analyzing the color channels of many fire pixels, it has been noticed that fire color patterns have the $\mathrm{Y}$ values that are greater than the

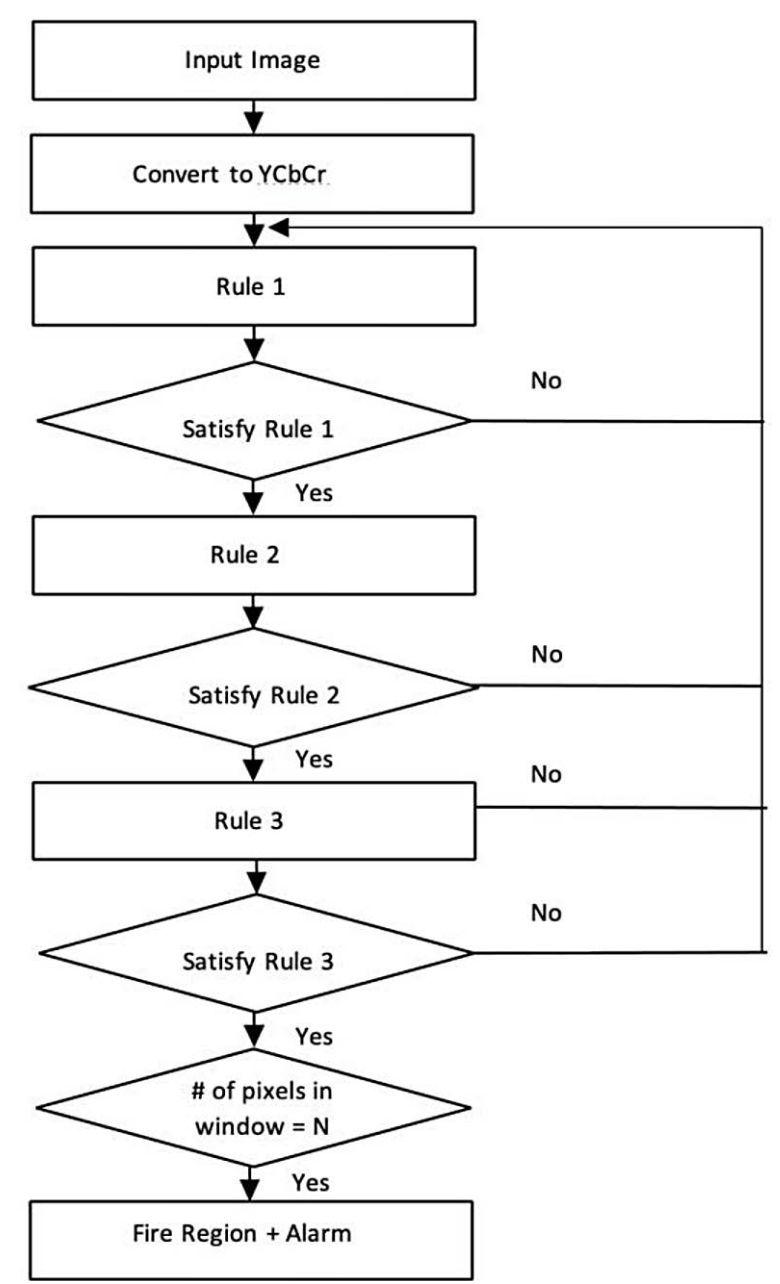

Fig. 5. Flowchart of the proposed method based on the $\mathrm{YCbCr}$ color model 


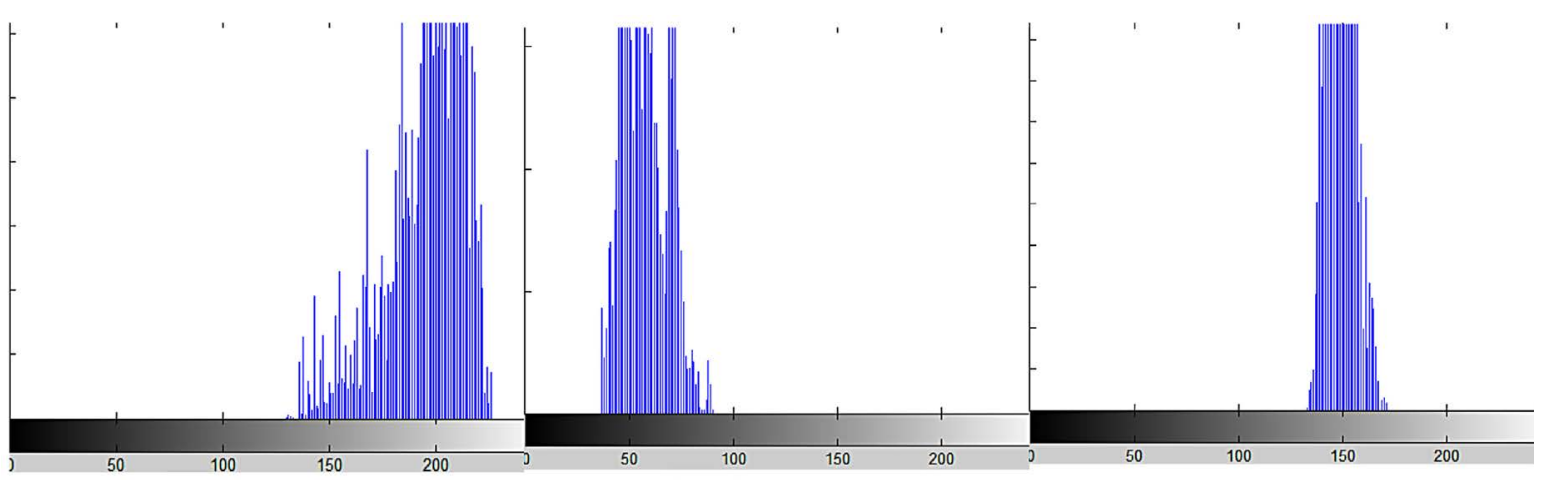

(a) Y channel

(b) $\mathrm{Cb}$ channel

(c) $\mathrm{Cr}$ channel

Fig. 6. Histogram of fire region

$\mathrm{Cb}$, and $\mathrm{Cr}$ values that are greater than $\mathrm{Cb}$. This can be observed from the histograms (Fig. 6) of $\mathrm{Y}, \mathrm{Cb}$ and $\mathrm{Cr}$ of a fire region, respectively.

[11] observed this characteristic and mentioned that it was verified over countless experiments with images containing fire regions and formulated as the following rule:

$$
F(x, y)=\left\{\begin{array}{c}
1, \text { if } Y(x, y) \geq C b(x, y) \text { and } \operatorname{Cr}(x, y) \geq \operatorname{Cb}(x, y) \\
0, \text { otherwise }
\end{array}\right.
$$

This can be considered as the first rule for detecting fire using the $\mathrm{YCbCr}$ color system. Fig. 7 shows the result of applying the first rule on some fire images from the considered dataset.

The first rule of detecting fire pixels operates efficiently when the background of the scene is dark; however, it misclassifies in the cases where the scene's background is bright.

\section{Rule 2: Difference between the $\mathrm{Cb}$ and Cr Components of Fire Pixels}

It can be noticed from Figure 8, that there is a significant difference between the $\mathrm{Cb}$ and $\mathrm{Cr}$ components of fire pixels. The $\mathrm{Cb}$ component is mainly "black" while the Cr component is mainly "white". This fact can be described as the following [7]:

$$
F(x, y)=\left\{\begin{array}{c}
1, \text { if }|\mathrm{Cb}(\mathrm{x}, \mathrm{y})-\operatorname{Cr}(x, y)| \geq \tau \\
0, \text { otherwise }
\end{array}\right.
$$

Where the value of the threshold $(\tau)$ is observed from the established receiver operating characteristics (ROC) curve. It is obtained by experimenting different values of $\tau$ ranging from 1 to 100 . The fire-pixel regions are manually segmented from each image. The true positive is

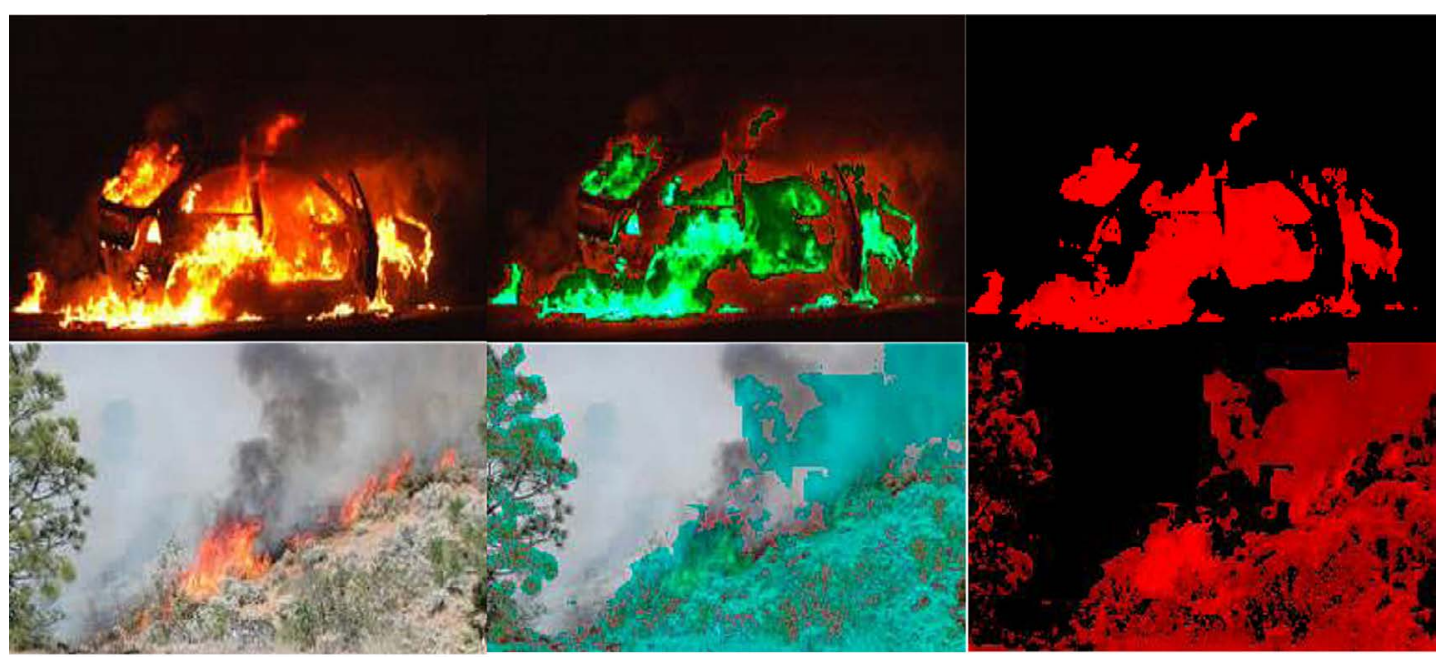

(a) Original fire images

(b) Results of applying the first rule on (a) (c) Results of applying the first rule on (a)

Fig. 7. Results of applying the first rule 


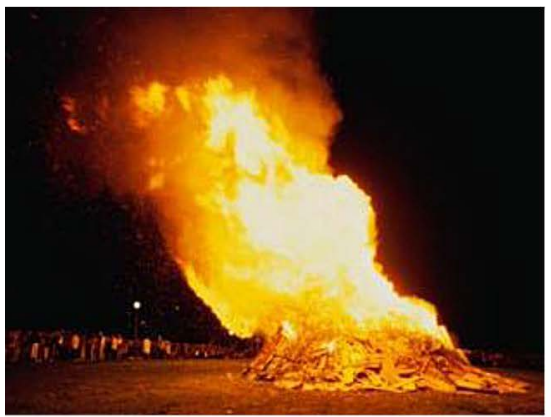

(a) Original image

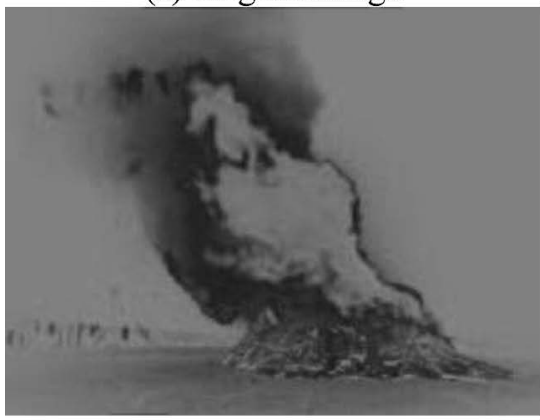

(c) $\mathrm{Cb}$ channel of (a)

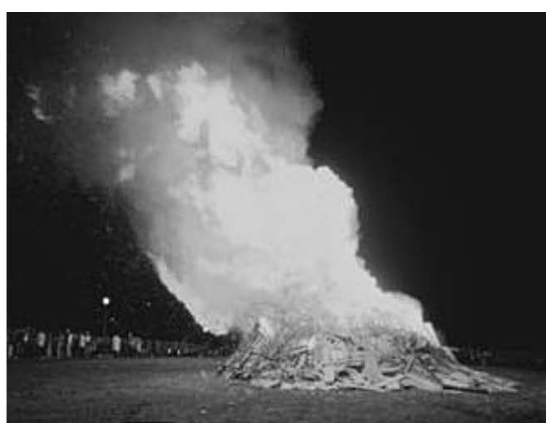

(b) Y channel of (a)

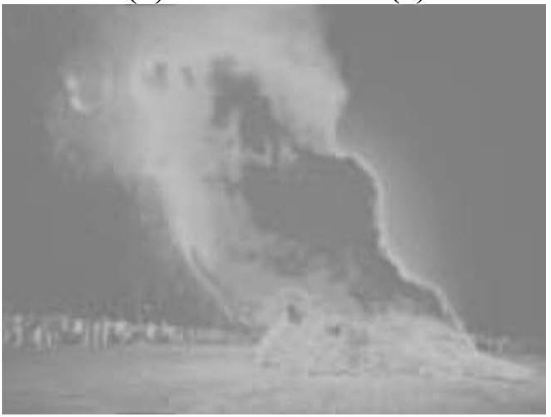

(d) Cr channel of (a)

Fig. 8. $\mathrm{Y}, \mathrm{Cb}$ and $\mathrm{Cr}$ channels

defined as the decision when an image contains a fire, and false positive is defined as the decision when an image contains no fire but is classified as having fire. According to the research conducted by [7], the ROC curve consisted of 100 data points corresponding to different $\tau$ values, the corresponding correct-detection and falsealarm rates are computed and recorded. They determined the $\tau$ value to be 40 after carrying out the analysis on a set of 1000 images. [11] also applied the same method on 100 images and determined $\tau$ as being 70 . In this work, different $\tau$ values ranging from 40 to 90 are tested on 50 images and it was observed that the best value for detecting the fire pixels was 70 .

By applying the difference between the $\mathrm{Cb}$ and $\mathrm{Cr}$ components of the fire pixels (thresholding by $\tau=70$ ) on some images from the dataset, the result of detecting the fire pixels is shown in [11].
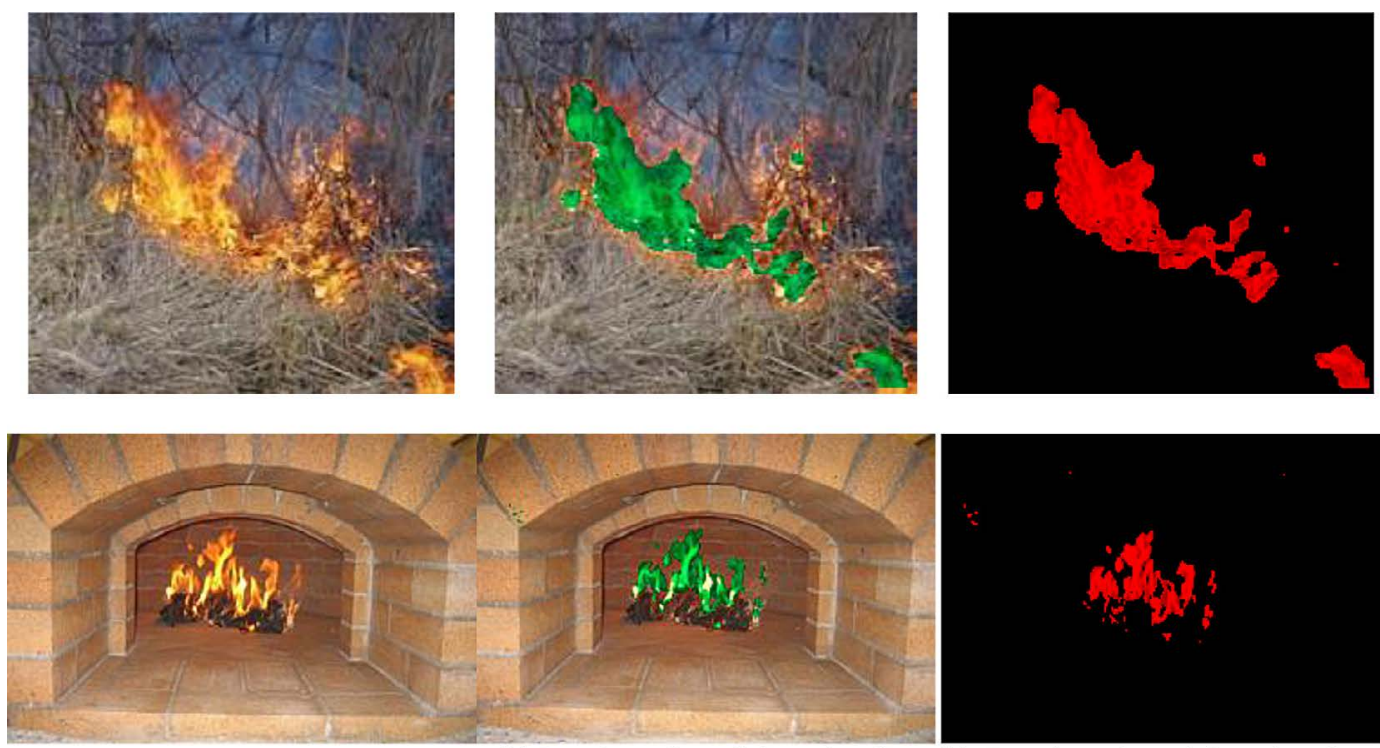

(a) Original fire images

(b) Results of applying the first (c) Results of applying the first rule on (a) rule on (a)

Fig. 9. Results of applying the first rule 
The thresholding rule is successful in detecting the fire pixels, but also misclassifies the fire-like pixels. That could result in increasing the false alarm rate in cases where no fire is present.

\section{Rule 3: YCbCr Clusters}

From the histogram analysis of fire regions, which are manually segmented, threshold values for the $Y$, $\mathrm{Cb}$ and $\mathrm{Cr}$ channels are defined. That has been accomplished by observing the histogram of thefire pixels for 50 images and recording the values of randomly selected fire pixels into a look-up table (Table 1). The look-up table was then converted into a graph and was divided into clusters (Fig. 10). After clustering, it can be observed that to consider a certain pixel as a fire pixel, it must have certain $\mathrm{YCbCr}$ value ranges for its pixels. The $\mathrm{Y}$ value was mostly less than 145 or greater than 170 , the $\mathrm{Cb}$ was less than or equal to 120 and greater than or equal to 50, and the $\mathrm{Cr}$ channel had various values that were limited between 120 and 220. This condition is formulated by:

$$
F(x, y)=\left\{\begin{array}{c}
1, \text { if } \mathrm{Y}(\mathrm{x}, \mathrm{y}) \geq 170 \mid \mathrm{Y}(\mathrm{x}, \mathrm{y})<145 \text { and } \operatorname{Cb}(x, y) \leq 120 \\
\operatorname{Cb}(x, y) \geq 50 \text { and } \operatorname{Cr}(x, y)>120 \\
\operatorname{Cr}(x, y)<220 \\
0, \text { otherwise }
\end{array}\right.
$$

The result of applying that rule on some of the dataset images is shown in Figure 11. Although it detects the fire-like pixels, it is effective in detecting the fire regions and especially the flame edges of fire regions.

\section{Results and Discussion of the $\mathrm{YCbCr}$ Algorithm}

As it is unlikely to have a single pixel in an image to represent a fire region, for that a window of n-by-n pixels is used as a regulation to consider a certain region as a fire region. In

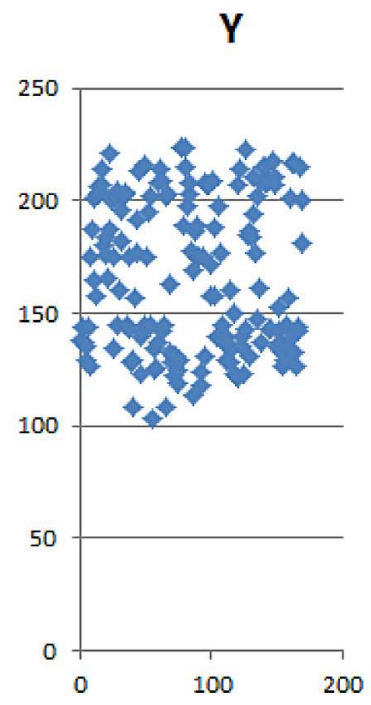

(a) Clusters of $\mathrm{Y}$

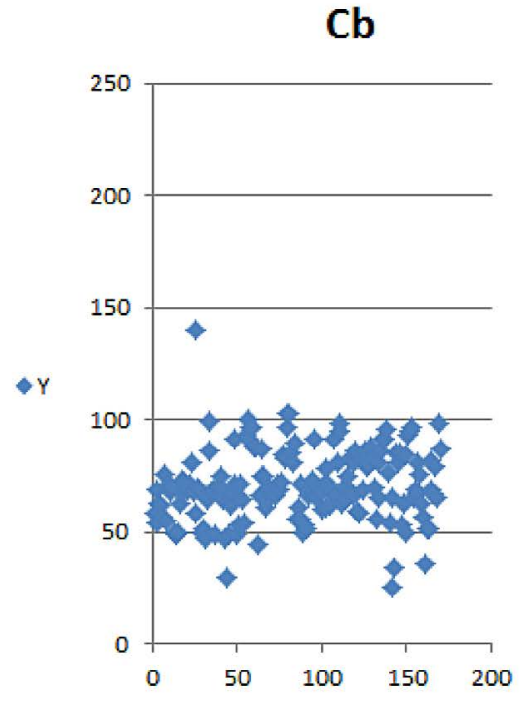

(b) Clusters of $\mathrm{Cb}$

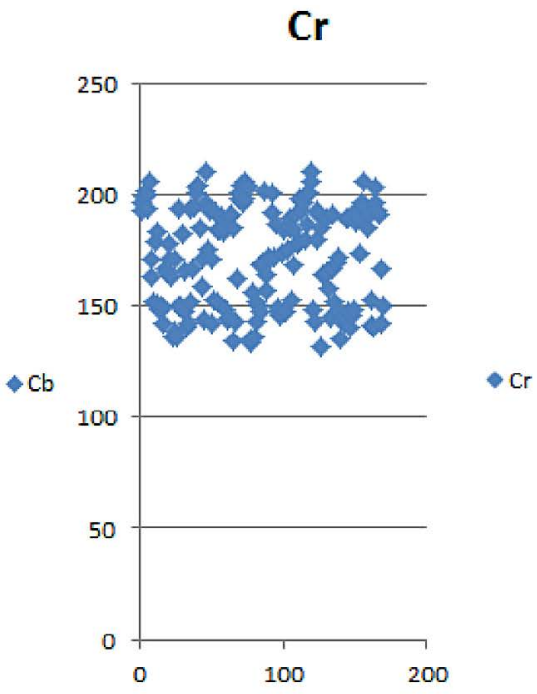

(c) Clusters of $\mathrm{Cr}$

Fig. 10. Clusters of $\mathrm{Y}, \mathrm{Cb}$ and $\mathrm{Cr}$

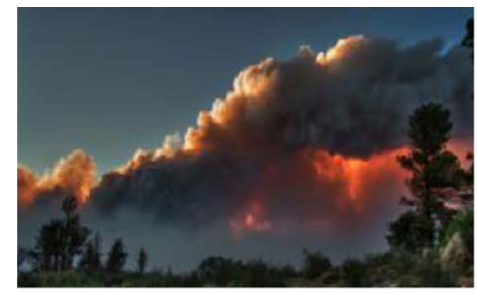

(a) Original fire image

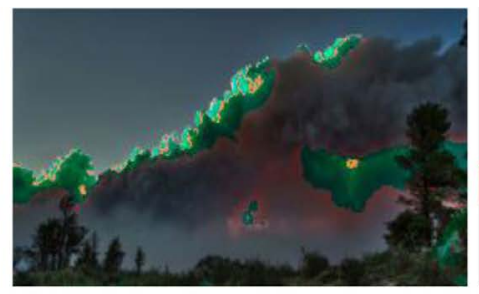

(b) Result of applying the third rule on (a)

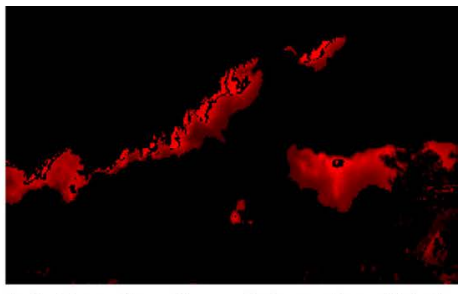

(c) Result of applying the third rule on (a)

Fig. 11. Result of applying the third rule 
Table 1. Values of random $\mathrm{Y}, \mathrm{Cb}$ and $\mathrm{Cr}$ pixels from 50 fire images

\begin{tabular}{|c|c|c|c|c|c|c|c|c|c|c|c|}
\hline$Y$ & $\mathrm{Cb}$ & $\mathrm{Cr}$ & $Y$ & $\mathrm{Cb}$ & $\mathrm{Cr}$ & $Y$ & $\mathrm{Cb}$ & $\mathrm{Cr}$ & $Y$ & $\mathrm{Cb}$ & $\mathrm{Cr}$ \\
\hline 138 & 58 & 196 & 160 & 51 & 182 & 126 & 97 & 183 & 186 & 56 & 164 \\
\hline 144 & 54 & 193 & 182 & 47 & 166 & 137 & 97 & 185 & 189 & 50 & 157 \\
\hline 137 & 69 & 199 & 196 & 64 & 147 & 208 & 88 & 148 & 175 & 53 & 172 \\
\hline 129 & 63 & 202 & 202 & 86 & 140 & 214 & 88 & 144 & 175 & 51 & 171 \\
\hline 135 & 62 & 200 & 204 & 99 & 142 & 209 & 44 & 145 & 124 & 66 & 201 \\
\hline 144 & 57 & 194 & 203 & 68 & 152 & 142 & 66 & 190 & 118 & 69 & 192 \\
\hline 127 & 76 & 206 & 145 & 69 & 194 & 145 & 75 & 191 & 175 & 73 & 172 \\
\hline 175 & 55 & 171 & 175 & 49 & 167 & 108 & 87 & 185 & 131 & 91 & 187 \\
\hline 187 & 71 & 163 & 143 & 70 & 195 & 202 & 75 & 134 & 208 & 68 & 147 \\
\hline 201 & 70 & 152 & 128 & 70 & 203 & 203 & 61 & 143 & 207 & 65 & 149 \\
\hline 165 & 68 & 179 & 108 & 75 & 201 & 163 & 69 & 162 & 207 & 70 & 146 \\
\hline 158 & 70 & 183 & 129 & 64 & 204 & 133 & 70 & 198 & 172 & 68 & 174 \\
\hline 203 & 50 & 151 & 157 & 69 & 185 & 128 & 66 & 201 & 158 & 60 & 184 \\
\hline 206 & 49 & 148 & 177 & 47 & 171 & 131 & 69 & 204 & 209 & 71 & 147 \\
\hline 204 & 50 & 150 & 192 & 30 & 159 & 122 & 68 & 196 & 188 & 61 & 147 \\
\hline 214 & 63 & 142 & 213 & 49 & 144 & 131 & 66 & 198 & 158 & 78 & 175 \\
\hline 207 & 73 & 149 & 140 & 62 & 196 & 119 & 71 & 206 & 140 & 71 & 189 \\
\hline 180 & 67 & 167 & 123 & 67 & 210 & 125 & 69 & 204 & 140 & 62 & 185 \\
\hline 184 & 72 & 165 & 142 & 91 & 175 & 129 & 72 & 203 & 198 & 68 & 153 \\
\hline 176 & 71 & 171 & 145 & 71 & 193 & 224 & 84 & 133 & 177 & 91 & 168 \\
\hline 166 & 69 & 178 & 216 & 49 & 142 & 224 & 83 & 135 & 138 & 66 & 184 \\
\hline 187 & 71 & 163 & 175 & 53 & 171 & 189 & 103 & 156 & 145 & 81 & 191 \\
\hline 221 & 81 & 136 & 195 & 71 & 153 & 224 & 97 & 136 & 137 & 98 & 178 \\
\hline 200 & 68 & 139 & 145 & 64 & 192 & 215 & 103 & 143 & 140 & 95 & 179 \\
\hline 175 & 58 & 171 & 202 & 54 & 152 & 198 & 85 & 152 & 129 & 63 & 198 \\
\hline 134 & 140 & 136 & 103 & 93 & 184 & 203 & 81 & 150 & 136 & 70 & 193 \\
\hline 202 & 70 & 150 & 134 & 100 & 184 & 208 & 90 & 147 & 128 & 64 & 196 \\
\hline 145 & 69 & 194 & 125 & 91 & 190 & 178 & 56 & 168 & 160 & 75 & 180 \\
\hline 205 & 50 & 150 & 114 & 71 & 202 & 169 & 61 & 169 & 134 & 83 & 200 \\
\hline 150 & 68 & 187 & 211 & 65 & 145 & 207 & 50 & 148 & 127 & 82 & 203 \\
\hline 123 & 66 & 201 & 177 & 81 & 167 & 135 & 84 & 192 & 144 & 67 & 193 \\
\hline 122 & 81 & 210 & 147 & 86 & 191 & 153 & 93 & 188 & 142 & 79 & 191 \\
\hline 121 & 86 & 206 & 202 & 91 & 152 & 142 & 95 & 191 & 215 & 65 & 142 \\
\hline 207 & 59 & 148 & 161 & 91 & 169 & 137 & 97 & 174 & 181 & 98 & 167 \\
\hline 214 & 58 & 143 & 137 & 96 & 172 & 127 & 65 & 189 & 200 & 87 & 150 \\
\hline 140 & 83 & 180 & 208 & 77 & 147 & 133 & 69 & 196 & 127 & 82 & 203 \\
\hline 135 & 69 & 193 & 215 & 54 & 135 & 129 & 81 & 206 & 144 & 67 & 193 \\
\hline 123 & 86 & 191 & 208 & 25 & 145 & 145 & 67 & 194 & 142 & 79 & 191 \\
\hline 143 & 79 & 185 & 215 & 65 & 142 & 135 & 76 & 195 & 215 & 65 & 142 \\
\hline 223 & 83 & 132 & 208 & 34 & 145 & 157 & 63 & 185 & 181 & 98 & 167 \\
\hline 185 & 80 & 164 & 143 & 85 & 189 & 139 & 57 & 191 & 200 & 87 & 150 \\
\hline 186 & 88 & 164 & 209 & 81 & 147 & 201 & 36 & 153 & 139 & 57 & 191 \\
\hline 131 & 83 & 189 & 143 & 85 & 189 & 217 & 51 & 141 & 223 & 83 & 132 \\
\hline 184 & 70 & 165 & 218 & 53 & 140 & 217 & 51 & 140 & 185 & 80 & 164 \\
\hline 194 & 56 & 158 & 211 & 63 & 146 & 133 & 69 & 196 & 215 & 65 & 152 \\
\hline
\end{tabular}


addition, by combining the three previously mentioned rules of detecting a fire pixel using $\mathrm{YCbCr}$ based system, Figure 12 and 13 illustrate the effect of each rule and the efficiency of all rules applied together on a certain image.

In order to evaluate the performance of the proposed algorithm, the dataset comprising 70 images which contain fire regions and 70 images containing the images with no fire regions and fire-like regions, was employed. Table 2 presents the confusion matrix for the predictions of the proposed algorithm.

$$
\text { accuracy }=\frac{\text { correct predictions }}{\# \text { of images }} \times 100 \%=\frac{120}{140} \times 100 \%=85.7 \%
$$

Table 2. Confusion matrix for the $\mathrm{YCbCr}$ proposed method

\begin{tabular}{|c|c|c|}
\hline & Fire & No fire \\
\hline Fire & 70 & 0 \\
\hline No fire & 20 & 50 \\
\hline
\end{tabular}

The algorithm was able to detect $100 \%$ of the fire images in the provided dataset as containing fire, but for non-fire images its accuracy was $71.4 \%$. The result gives a good impression on using the proposed $\mathrm{YCbCr}$ based algorithm on

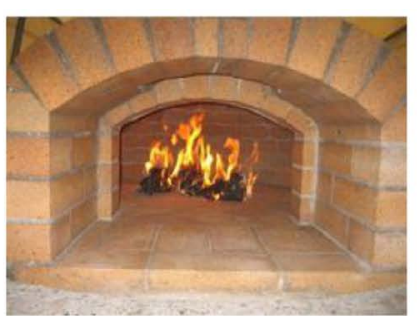

(a) Original fire image

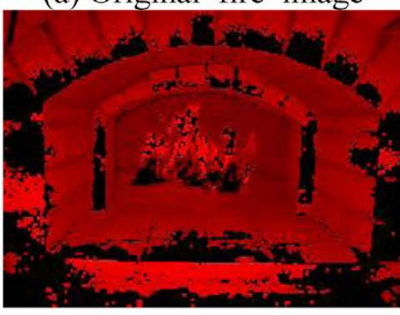

(d) Result of applying rule 3
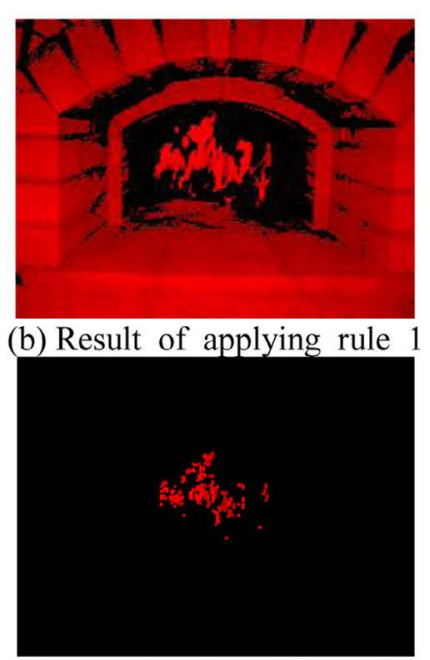

(d) Result of applying all rules

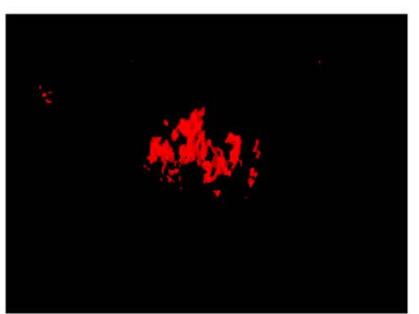

(c) Result of applying rule 2

Fig. 12. Result of applying the rules

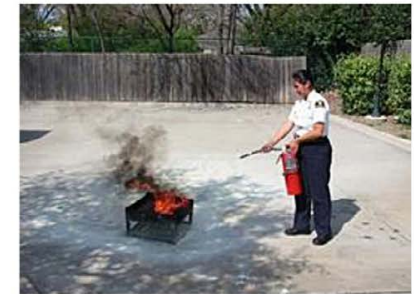

(a) Original fire image

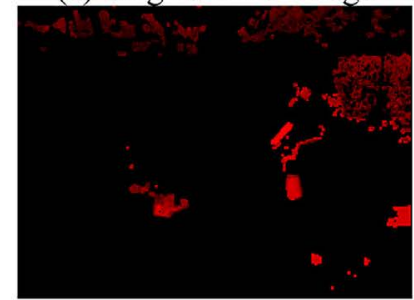

(d) Result of applying rule 3

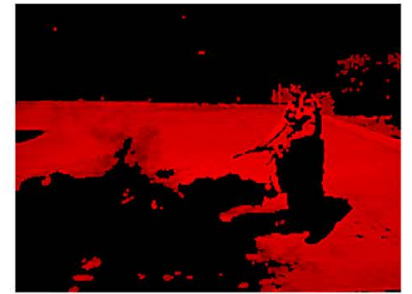

(b) Result of applying rule 1

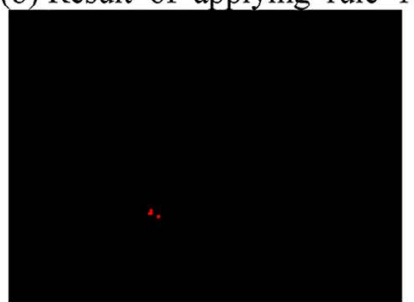

(d) Result of applying all rules

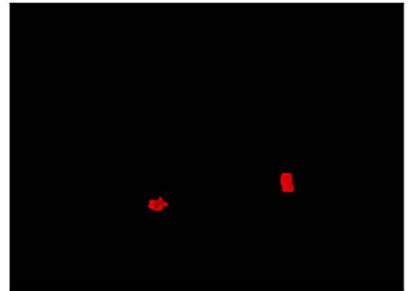

(c) Result of applying rule 2

Fig. 13. Result of applying the rules 
promoting the safety issues. On the other hand, it gives false alarms in almost $28.6 \%$ with respect to the investigated dataset which will also have effects on other sides, as the expenses of evacuating. Moreover, it should be considered that the achieved accuracy included applying the algorithm on indoor and outdoor data set images with different environmental and weather conditions. In addition, the accuracy was achieved by only applying three simple and efficient rules. That requires less computation complexity which enables to use this algorithm in real-time fire detection.

\section{The HSV Based Method}

In this section, the details of detecting fire regions based on HSV color model are presented. Figure 14 indicates the flow chart of the approach

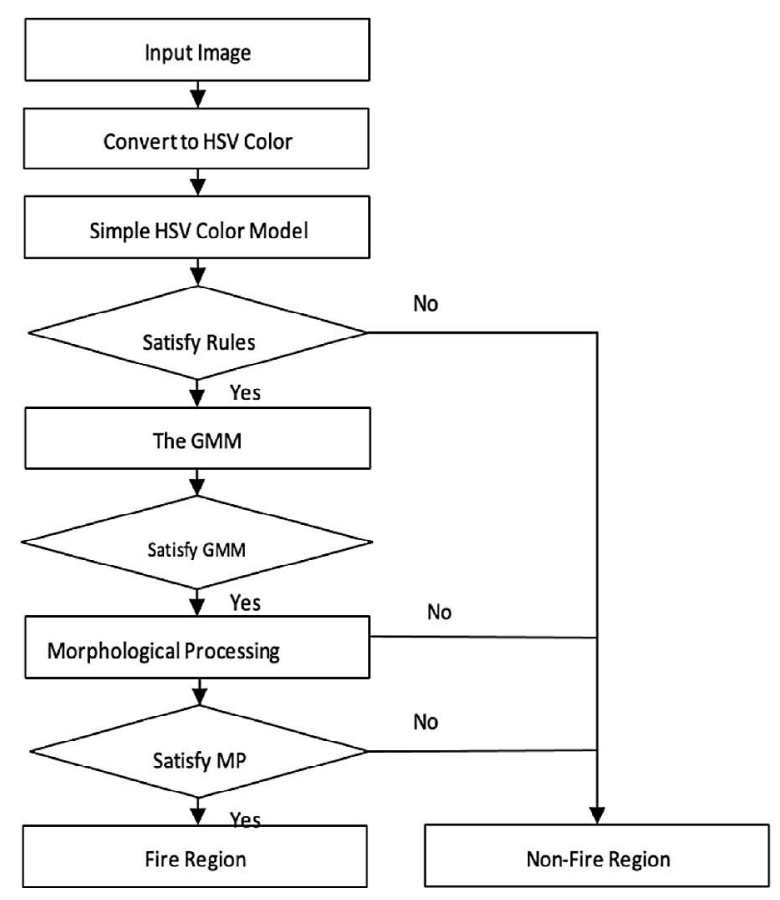

Fig. 14. Flowchart of the proposed method based on the HSV color model in which the input RGB image is firstly converted to HSV color space. Next, the color values of each pixel are checked according to three HSV decision rules. The pixels satisfying the decision rules are further checked by Gaussian mixture model (GMM). Finally, some morphological postprocessing techniques are used on the detection result. The overall accuracy the dataset of the 140 images is $85.7 \%$.

\section{The Three Decision Rules of the HSV Color Space Based Algorithm}

Among various color systems, HSV (Hue, Saturation, Value) color model is very suitable for human interpretation, since hue, saturation and values components are intimately related to the way in which human beings perceive color. Empirically, the burning fire generally displays some obvious color features. For instance, the typical flame shows the white kernel, as well as red and yellow colors around the kernel.

On the basis of human interpretations of fire features, it is reasonable to assume that the flame color belongs to certain ranges of HSV components. In order to obtain the threshold values to separate flame color, the histograms shown in Fig. 15 were generated for fire pixels of HSV components. It can be concluded from these histograms that the fire pixels usually take much lower $\mathrm{H}$ values, but relatively higher $\mathrm{S}$ and $\mathrm{V}$ values. After a lot of experiments, the following decision rules to segment fire by HSV color space were defined:

Rule 1: $0 \leq H_{(x, y)} \leq 0.35 \cup 3.0 \leq H_{(x, y)} \leq 3.14$

Rule 2: $0.3 \leq S_{(x, y)} \leq 1.0$

Rule 3: $0.8 \leq V_{(x, y)} \leq 1.0$

As shown in Figure 16, the above inequalities are applied to generate some binary maps which indicate whether the pixel located at

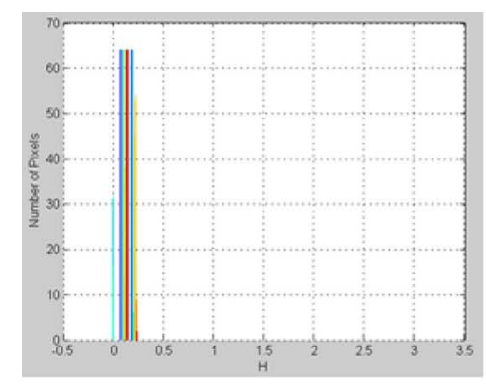

(a) $\mathrm{H}$ component histogram

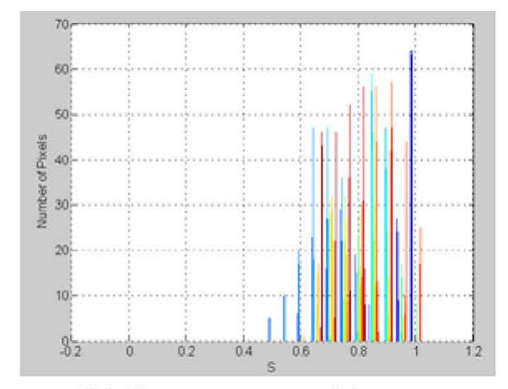

(b) $\mathrm{S}$ component histogram

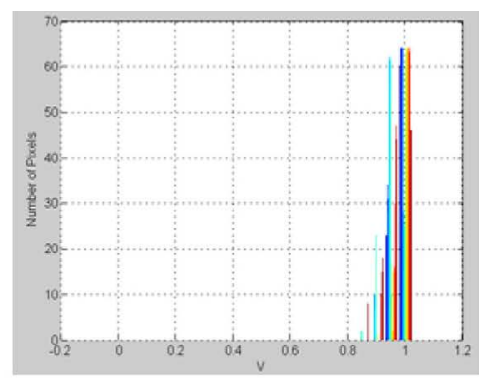

(c) V component histogram

Fig. 15. The fire pixels histograms 
spatial location $(\mathrm{x}, \mathrm{y})$ is classified as fire (binary value of 1 ) or non-fire (binary value of 0 ) pixel. Specially, Figure 16(a) is the input image of hay fire; Figure 16(b) shows the fire detection result after using Rule 1, which not only segments the fire region but also incorrectly takes the color of hay as the fire pixels. After combining Rule 1 and Rule 2, the fire detection result provided in Figure 16(c) has been improved to some extent, although the result is still not satisfactory. When combining three decision rules together, a good visual result is obtained in Figure 16(d) in which almost all the fire regions in Figure 16(a) have been segmented correctly.

Although the above three decision rules show effectiveness in separating fire regions in some images, just using those simple inequalities tend to fail distinguish fire and objects with firelike colors. This is because those three decision rules only define rough color ranges for flame, and some other fire-like colors are included in

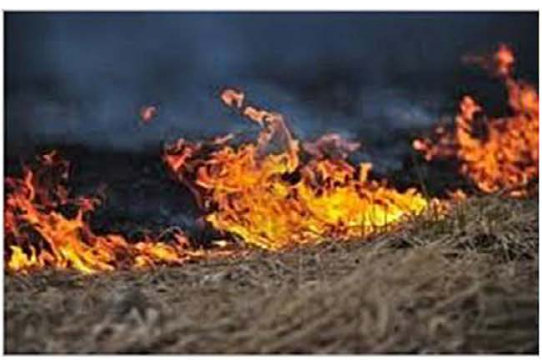

(a) Original image

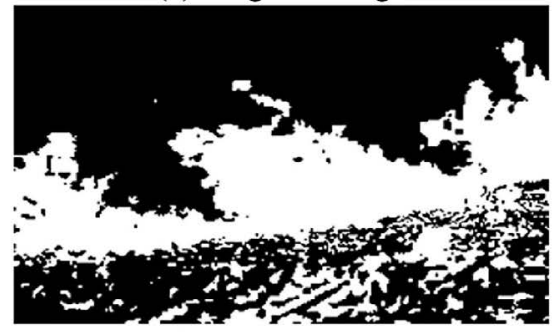

(c) segmentation using rules (1) and (2) those ranges as well. Figure 17(a) shows an image with reddish color, while Figure 17(b) is the segmentation result using above three rules. Apparently, all the fire-like colors in Figure (a) are mistakenly taken as fire regions.

\section{Gaussian Mixture Model Based Fire Detection}

Since using only simple mathematical formulations in HSV color space to differentiate between the fire and fire-like colors is impossible, it is necessary to explore more accurate fire color models to represent the fire pixels distribution. As shown in Figure 18, 1024 fire pixels segmented manually from fire images are plotted in 3D space. In order to more precisely represent the 3D shape of the point cloud in Figure 18, Gaussian mixture model (GMM) is applied in this work, thus the pixel the color of which is within the range of GMM distribution model can be regarded as a candidate flame pixel.

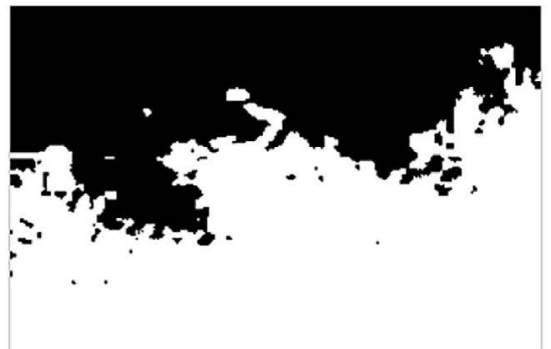

(b) segmentation using only rule (1)

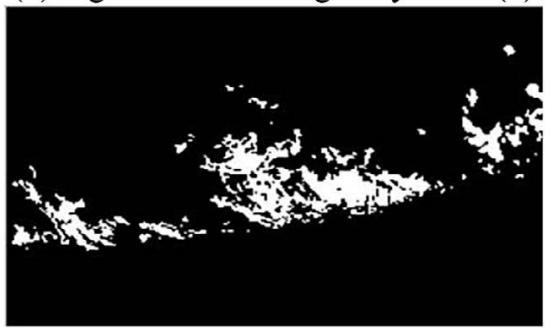

(d) segmentation using rules (1), (2) and (3)

Fig. 16. Fire pixels segmentation

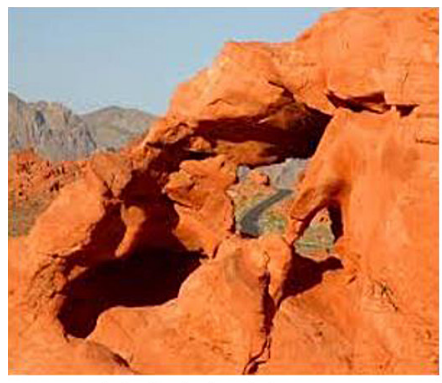

(a) Original image

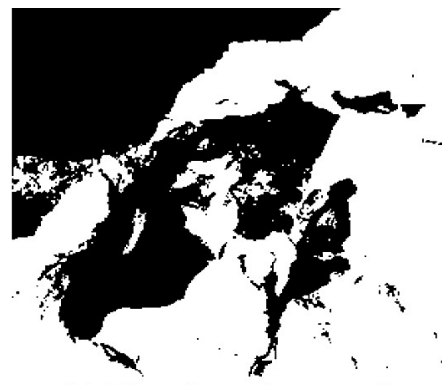

(b) The detection result

Fig. 17. Fire-like color detection using decision rules 


\section{Gaussian Mixture Model}

Assume there are M single Gaussian models that describe sampled fire pixels, and each single Gaussian model takes the proportion as $a_{j}(1 \leq j \leq M)$, the probability density function (p.d.f) of an observation $x_{i}$ belonging to the defined GMM can be represented by the following equation:

$$
p\left(x_{i}\right)=\sum_{j=1}^{M} a_{j} N_{j}\left(x_{i} ; \mu_{j}, \Sigma_{j}\right)
$$

where: $a_{j}(1 \leq j \leq M)$ is the mixing parameter, $N_{j}\left(x_{i} ; \mu_{j}, \Sigma_{j}\right)$ is the $j_{\text {th }}$ single Gaussian model.

They are expressed as:

$$
\begin{gathered}
\sum_{j=1}^{M} a_{j}=1 \\
N_{j}\left(x_{i} ; \mu_{j}, \Sigma_{j}\right)=\frac{1}{\sqrt{(2 \pi)^{m}\left|\Sigma_{j}\right|}} \exp \left[-\frac{1}{2}\left(x_{i}-\mu_{j}\right)^{T} \Sigma_{j}^{-1}\left(x-\mu_{j}\right)\right]
\end{gathered}
$$

where: $\mu_{j}$ is the mathematical expectation of the $j_{t h}$ model, $\Sigma_{j}$ is the covariance of the $j_{t h}$ model. In order to describe fire pixels distribution in 3D space by the GMM, the main premise is to ascertain the unknown parameters $M, a_{j}, \mu_{j}$, and $\Sigma_{j}$. The number $(M)$ of single Gaussian models can be manually assigned or obtained by the trial-and-error method. In this work, eight single Gaussian models were chosen after multiple experiments. For the other three unknown parameters, they can be estimated by the expectation maximization (EM) algorithm discussed in the following section.

\section{Estimating Parameters Using EM Algorithm}

There are various approaches that can be used for determining the parameters of the GMM from a set of initial data Hirsch (2004). In this work, the expectation maximization (EM) algorithm is deployed for the GMM parameters estimation.

Regarding to the GMM in equation (10), assume that $\theta_{j}=\left(a_{j}, \mu_{j}, \Sigma_{j}\right)$ and $\Phi=\left(\theta_{1}, \ldots \theta_{M}\right)^{T}$, the loglikelihood for the data set $X=\left\{x_{i} \mid 1 \leq i \leq N\right\}$ is given by:

$$
l((X \mid \Phi))=\log \prod_{i=1}^{N} \sum_{j=1}^{M} a_{j} N_{j}\left(X ; \mu_{j}, \Sigma_{j}\right)=\sum_{i=1}^{N} \log \sum_{j=1}^{M} a_{j} N_{j}\left(x_{i} ; \mu_{j}, \Sigma_{j}\right)
$$

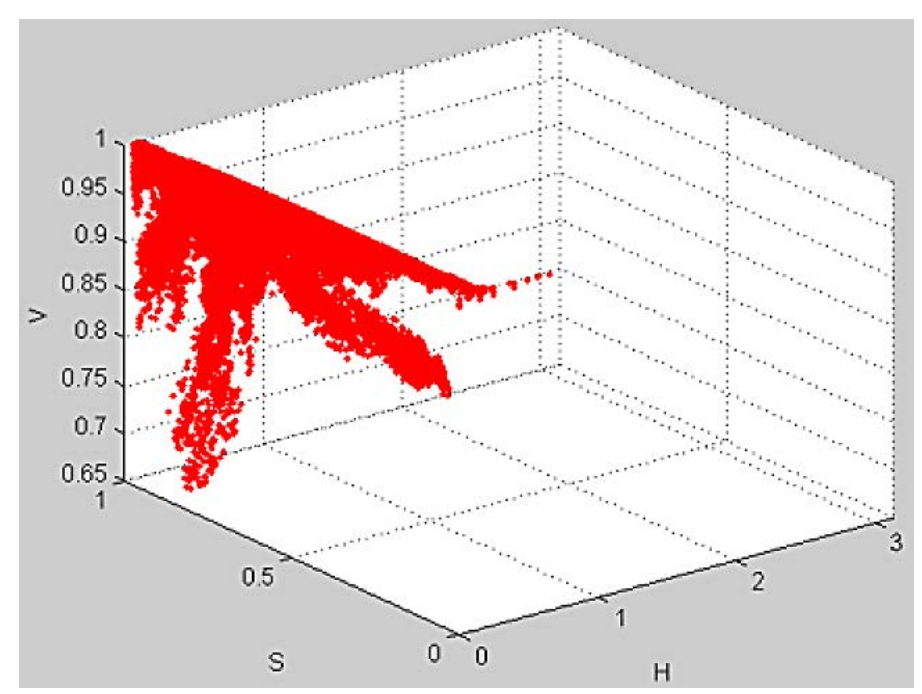

Fig. 18. 3D point cloud of sampled fire pixels 
When the value of $\left|l(X \mid \Phi)-l(x \mid \Phi)^{\prime}\right|$ is continually minimized, such that it is less than a threshold value $\varepsilon$, for example $10^{-5}$ the GMM is converged. However, the EM algorithm should start with some initial guess for the parameters of the GMM. In this work, initialization of the mathematical expectation $\mu_{j_{0}}$ and the covariance $\Sigma_{j_{0}}$ of the model through K-means clustering method, and the mixing parameter $a_{j_{0}}$ was computed based on the clustering result. According to the initial guess for the GMM unknown parameters, the posterior probability for $a_{j}(1 \leq j \leq M)$ is computed as:

$$
\beta_{j}=E\left(\left(a_{j} \mid x_{i} ; \Phi\right)\right)=\frac{a_{j} N_{j}\left(x_{i} ; \Phi\right)}{\sum_{j}^{M} a_{j} N_{j}\left(x_{i} ; \Phi\right)}, 1 \leq i \leq N, 1 \leq j \leq M
$$

After computing the posterior probability $\beta_{j}$, the values of parameters $a_{j}, \mu_{j}, \Sigma_{j}$ are be updated by:

$$
\begin{gathered}
a_{j}^{\prime}=\frac{\sum_{i}^{N} \beta_{i j}}{N} \\
\mu_{j}^{\prime}=\frac{\sum_{i=1}^{N} \beta_{i j} x_{i}}{\sum_{i=1}^{N} \beta_{i j}} \\
\Sigma_{j}^{\prime}=\frac{\sum_{i=1}^{N} \beta_{i j}\left(x_{i}-\mu_{j}^{\prime}\right)\left(x_{i}-\mu_{j}^{\prime}\right)^{T}}{\sum_{i=1}^{N} \beta_{i j}}
\end{gathered}
$$

Using the updated parameters, the value of could be computed iteratively until the GMM is converged. Then, whether one pixel belongs to fire region of the image being processed can be determined by computation of its probability with (10).

\section{Morphological Post-Processing}

The approach of combining the HSV decision rules and the GMM works relatively well in distinguishing the fire and fire-like colors, but some issues still exist. For example, some isolated fire-like pixels may be still incorrectly taken as the fire pixels (Figure 21(c)), and not all fire pixels are segmented from the image (Figure 20(c)). In order to further enhance the algorithm, morphological processing techniques are adopted to improve the fire detection results. Firstly, the number of the fire pixels in each connected component is calculated. Only when the number of pixels is greater than the predefined threshold like 20, can the component be regarded as the fire region. In addition, morphological opening and closing operations can be utilized to fill some possibly existing holes in the detected fire regions.

\section{HSV model Experimental Results}

The HSV color space based fire detection mainly involves three parts: simple HSV color decision rules, GMM and morphological postprocessing. Before using GMM, manual segmentation of fire samples and considering them as training data. As shown in Figure 20, 1024 fire pixels are applied to estimate the unknown parameters of the GMM by the EM algorithm.

Figure 20 shows an example of fire detection using the proposed algorithm, in which Figure 20(a) is the input fire image, Figure 20(b) is the detection result under three decision rules of HSV color model. Clearly, the detection result by the simple HSV color model is not satisfied, since a lot of fire-like pixels such as the color of smoke and red house are incorrectly taken as flames. Figure 20(c) indicates the fire regions detected by the HSV color model and GMM, while Figure 20(d) is the final result after the morphological post-processing. It can be seen that GMM is more robust in distinguishing fire and fire-like colors than the decision rules of the HSV color model. Especially, the relatively good result was obtained after filling holes in the fire regions.

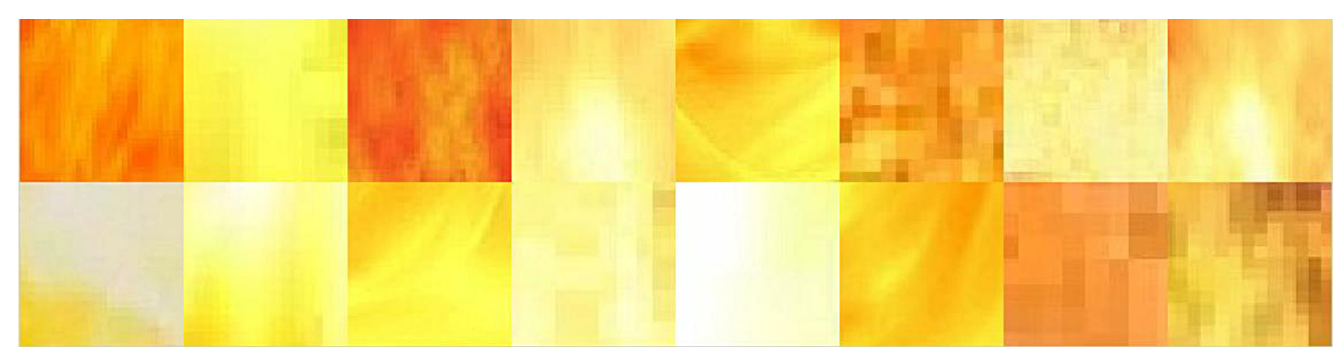

Fig. 19. Training samples segmented from real fire images 


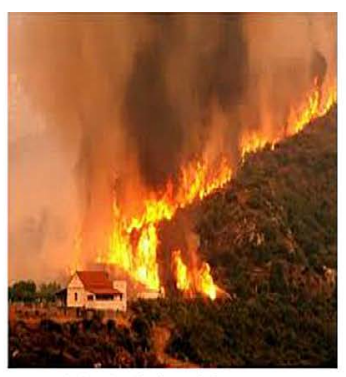

(a) Original image

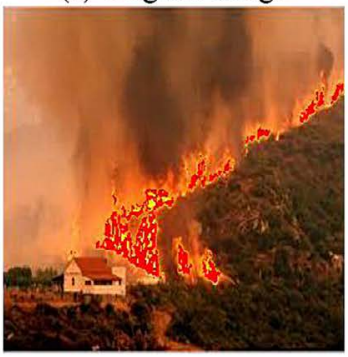

(c) fire pixels detected by HSV color model and the GMM

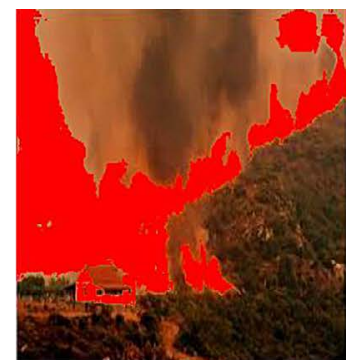

(b) Fire pixels detected by HSV color model

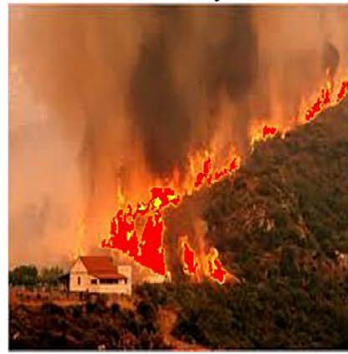

(d) the final detection result

Fig. 20. Fire regions segmentation

Further examples of successful detecting nonfire colors are shown in Figure 21, the original input image of Figure 21(a) contains the red and yellow color with high illumination, which has the high similarity with the fire color. Without doubt, the decision rules based on a simple HSV color model misclassifies many regions as the fire pixels which are shown as binary "1" in Figure 21(b). Even worse, there are still some isolated pixels that are detected as the fire pixels by the trained GMM (Fig. 21(c)). After adding another

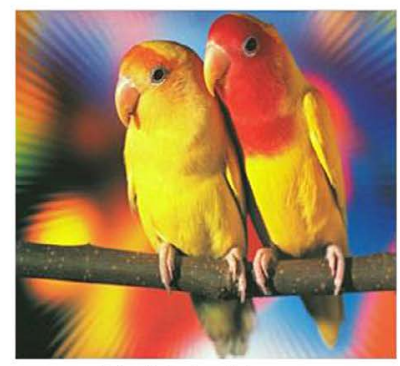

(a) Original image

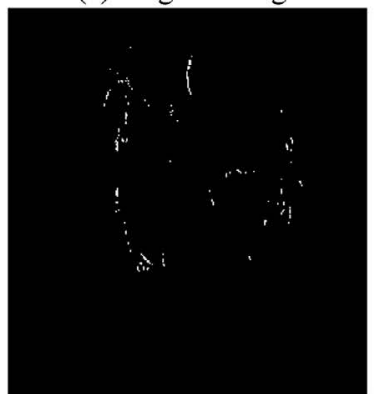

(c) Segmentation result by HSV color model and the GMM constraint that the number of pixels in the fire regions should be greater than the predefined threshold, the correct classification result is successfully gained, which is shown in Figure 21(d).

During the simulations, the algorithm was tested on real-world images that are taken from a random selection on Internet. As previously mentioned for the RGB and YCbCr color models, the collected images were divided into two groups, namely the fire and non-fire group, and each group had 70 images. In the fire group, it consisted of different types of

Fig. 21. Fire-like colors segmentation 
fires such as forest fire and residential fire. Besides, the images were captured at day time or night time to evaluate the algorithm performance under different lighting conditions. In the non-fire group, it consisted of the objects with fire-like colors such as red trunk and sun. (Table 3 ) shows the detection results of our algorithm on the fire and non-fire images, in which the first column is the sequence numbers of images in each group, and the " 1 " in the other two columns means fire alarming, but " 0 " represents no fire in the detected image.

As indicated by Table 3, the accuracies of the algorithm shown in (Table 4), in which the accuracy for fire group is much higher, $91.4 \%$, and the accuracy for the non-fire group is relatively lower, $80.0 \%$. The overall accuracy for both groups is $85.7 \%$. The relatively lower accuracy on non-fire images is mainly due to too many images in the non-fire group having fire-like colors.

\section{CONCLUSIONS}

This work presented three intelligent approaches to detect fires based on three different color models: RGB, $\mathrm{YCbCr}$ and HSV. The accuracy of approximately $86 \%$ was achieved by applying the three approaches to the data set consisting of 140 images, which had been captured indoor and outdoor under different environmental and weather conditions. In addition, each technique deployed three simple but efficient rules, which requires less computation complexity. These two advantages enable the algorithms to be used in real-time fire detection. The RGB and $\mathrm{YCbCr}$ methods were able to detect $100 \%$ of the cases on the fire image set. The HSV adopted the Gaussian mixture model (GMM). In turn, the expectation maximization (EM) algorithm presented the best results on the fire-free images by predicting $80 \%$

Table 3. The detection results on fire and non-fire images

\begin{tabular}{|c|c|c|c|c|c|c|c|c|}
\hline Number & Fire & Non-Fire & Number & Fire & Non-Fire & Number & Fire & Non-Fire \\
\hline 1 & 1 & 0 & 25 & 1 & 0 & 49 & 1 & 1 \\
\hline 2 & 1 & 1 & 26 & 1 & 0 & 50 & 1 & 0 \\
\hline 3 & 1 & 0 & 27 & 1 & 0 & 51 & 1 & 1 \\
\hline 4 & 1 & 1 & 28 & 1 & 1 & 52 & 1 & 0 \\
\hline 5 & 1 & 0 & 29 & 1 & 0 & 53 & 1 & 0 \\
\hline 6 & 1 & 0 & 30 & 1 & 0 & 54 & 1 & 0 \\
\hline 7 & 1 & 0 & 31 & 1 & 0 & 55 & 1 & 0 \\
\hline 8 & 1 & 1 & 32 & 1 & 0 & 56 & 1 & 0 \\
\hline 9 & 1 & 1 & 33 & 1 & 0 & 57 & 1 & 0 \\
\hline 10 & 1 & 0 & 34 & 1 & 1 & 58 & 1 & 0 \\
\hline 11 & 0 & 0 & 35 & 1 & 0 & 59 & 1 & 0 \\
\hline 12 & 1 & 0 & 36 & 1 & 0 & 60 & 1 & 0 \\
\hline 13 & 0 & 0 & 37 & 1 & 0 & 61 & 1 & 0 \\
\hline 14 & 1 & 1 & 38 & 1 & 0 & 62 & 1 & 0 \\
\hline 15 & 1 & 0 & 39 & 1 & 0 & 63 & 1 & 0 \\
\hline 16 & 1 & 0 & 40 & 1 & 0 & 64 & 1 & 0 \\
\hline 17 & 1 & 0 & 41 & 1 & 0 & 65 & 1 & 0 \\
\hline 18 & 1 & 1 & 42 & 1 & 0 & 66 & 1 & 0 \\
\hline 19 & 1 & 0 & 43 & 0 & 0 & 67 & 0 & 0 \\
\hline 20 & 1 & 1 & 44 & 1 & 0 & 68 & 1 & 0 \\
\hline 21 & 1 & 1 & 45 & 1 & 0 & 69 & 1 & 0 \\
\hline 22 & 1 & 1 & 46 & 1 & 0 & 70 & 1 & 0 \\
\hline 23 & 1 & 1 & 47 & 1 & 0 & & & \\
\hline 24 & 0 & 0 & 48 & 0 & 0 & & & \\
\hline
\end{tabular}

Table 4. The accuracies of utilizing the HSV color model

\begin{tabular}{|l|c|c|c|}
\hline \multicolumn{1}{|c|}{ Test data } & Correct detection & Total number & Accuracies \\
\hline Fire group & 64 & 70 & $91.4 \%$ \\
\hline Non-Fire group & 56 & 70 & $80.0 \%$ \\
\hline
\end{tabular}


of the images without fire. There was a trade-off between the accuracy of detecting the fire regions precisely and the probability of detecting the firelike regions. In order to improve the reliability of the presented techniques, dynamic characteristics of flame such as flame motion and geometry are to be integrated in the future.

\section{REFERENCES}

1. Yu C. Mei Z. Zhang X. A Real-Time Video Fire Flame and Smoke Detection Algorithm. Procedia Engineering. 2013;62:891-898. DOI: 10.1016/j. proeng.2013.08.140

2. Gong F., Li C., Gong W., Li X., Yuan X., Ma Y., Song T. A Real-Time Fire Detection Method from Video with Multifeature Fusion. Computational Intelligence and Neuroscience. 2019;1687-5265. DOI: 10.1155/2019/1939171

3. Töreyin B., Dedeoglu,Y., Gudukbay U., Cetin A. Computer Vision Based Method for Real-Time Fire and Flame Detection. Pattern Recognition Letters. 2005;27:49-58. DOI: 10.1016/j.patrec.2005.06.015

4. Appana D., Islam R., Khan S., Kim J. A VideoBased Smoke Detection Using Smoke Flow Pattern and Spatial-Temporal Energy Analyses for Alarm Systems. Information Sciences. 2017;418-419:91101, DOI:10.1016/j.ins.2017.08.001

5. Celik T. Fast And Efficient Method for Fire Detection Using Image Processing. ETRI Journal. 2010;6:881-890. DOI:10.4218/etrij.10.0109.0695

6. Hsu T., Pare S., Meena S., Jain D., Li D., Saxena A., Prasad M., Lin C. An Early Flame Detection System Based on Image Block Threshold Selec- tion Using Knowledge of Local and Global Feature Analysis. Sustainability. 2020;12(21):1-22. DOI:10.3390/su12218899

7. Celik T., Demirel H., Ozkaramanli H., Uyguroglu M. Fire Detection Using Statistical Color Model in Video Sequences. Journal of Visual Communication and Image Representation. 2007;18:176-158. DOI:10.1016/j.jvcir.2006.12.003

8. Yu Z., Xu Y., Yang X. Advances in multimedia modeling, Springer, Berlin Heidelberg. 2010;477-488.

9. Tong X., Li R., Ge L., Zhao L., Wang K. ANew Edge Patch with Rotation Invariance for Object Detection and Pose Estimation, Sensors. 2020;20(3):887. DOI: $10.3390 / \mathrm{s} 20030887$

10. Li Y., Wu W. Sequential Pattern Technology for Visual Fire Detection. Journal of Electronic Science and Technology. 2012;1:276-280. DOI:10.3969/j. issn.1674-862X.2012.03.014

11. Vipin V. Image Processing Based Forest Fire Detection. International Journal of Emerging Technology and Advanced Engineering. 2012;2:87-95. DOI:10.1155/2018/7612487

12. Valikhujaev Y., Abdusalomov A., Cho Y.I. Automatic Fire And Smoke Detection Method for Surveillance Systems Based on Dilated CNNs. Atmosphere. 2020;11(11):1241. DOI: 10.3390/atmos11111241

13. Hirsch R. Exploring colour photography: A complete guide. Laurence King, London, UK. 2004.

14. Yang M.H., Ahuja A. Gaussian mixture model for human skin color and its applications in image and video databases. Proceedings of Storage and Retrieval for Image and Video Databases Engineering (SPIE 99). 1999;3656:458-466.

15. Varma S., Behera V. Human Skin Detection Using Histogram Processing and Gaussian Mixture Model Based on Color Spaces. ICISS. 2017;116-120. 